\title{
Technikfolgen-Abschätzung: Entscheidungshilfe in einer komplexen Welt
}




\title{
Technikfolgen-Abschätzung: Entscheidungshilfe in einer komplexen Welt
}

\author{
Gunther Tichy
}

\section{Keywords}

Technikfolgen-Abschätzung, technology assessment, Riskokonzepte, risc concepts, Spezialisierung, specialisation, Entscheidungsvorbereitung, preparation of decison-making, expert knowledge, Expertenwissen, Interdisziplinarität, interdisciplinarity

\section{Zusammenfassung}

Selbst in der wissenschaftlichen Öffentlichkeit wird die Verhinderung bzw. Förderung von Techniken vielfach als Aufgabe von TA gesehen. Das wäre jedoch nicht bloß mit demokratischen Strukturen unvereinbar, es fehlte dafür auch die wissenschaftliche Basis. Die jeweiligen Vor- und Nachteile einer Technik können nicht wertneutral gegeneinander abgewogen werden, und die Konfliktparteien vertreten zumeist unterschiedliche Risikokonzepte, inkompatible Weltanschauungen und Zukunftsvisionen. Demgemäß kann TA eine politische Lösung solcher Konflikte nicht ersetzen, diese jedoch durch Herausarbeiten der jeweiligen Folgen unterstützen. Ein Bedarf dafür ergibt sich, weil unsere Welt nicht zuletzt durch die Kumulierung von Wissen komplexer geworden ist. Zunehmende Spezialisierung war die unvermeidliche Reaktion; sie ermöglichte zwar den beeindruckenden wissenschaftlichen Fortschritt der letzten Jahrzehnte, erschwert aber zugleich gesamthafte Beurteilungen, die zwangsläufig breites und fachübergreifendes Wissen erfordern. TA versucht diese Lücke im Wege interdisziplinärer Zusammenarbeit zu füllen, und Entscheidungsunterlagen überall dort aufzubereiten, wo sich ein und dasselbe Problem aus dem Blickwinkel unterschiedlich spezialisierter Experten unterschiedlich darstellt und zu unterschiedlichen Expertisen und Entscheidungsgrundlagen führt. Zentrale Aufgabe von TA-Experten als interdisziplinäre Generalisten ist daher die Verbesserung der Entscheidungsgrundlagen durch Vernetzung der unterschiedlichen, mit der jeweiligen Frage befassten Fachexperten. Die Arbeit diskutiert, zu welchen Fragen und auf welchen Gebieten TA solche Entscheidungshilfen bieten kann, und illustriert die Möglichkeiten an Hand einiger Arbeiten des österreichischen Instituts für Technikfolgen-Abschätzung.

(c) IT - Institut für Technikfolgen-Abschätzung der Österreichischen Akademie der Wissenschaften A-1030 Wien, Strohgasse 45/5, Fax: +43-I-710 9883 http://www.oeaw.ac.at/ita/ 


\begin{abstract}
The public and even scientists consider prevention and promotion of technologies as technology assessments' primary task. This view is incompatible with democracy and devoid of any scientific foundation. Evaluating the cost and benefits of a technology affords value judgements, and the disputing parties lack common risk concepts, world views and visions. Insofar TA cannot substitute a decision by elected politicians, but it can improve the policy decision by assessing likely consequences. Support of this type is needed as cumulating knowledge made our world increasingly complex. General specialisation and, in particular, specialisation of scientists was the unavoidable consequence. It enabled the impressive technical progress but hinders holistic assessments as they afford general interdisciplinary knowledge. TAs' task is to fill this gap and prepare decisions in all those cases where a problem presents itself differently to experts of different fields. The paper discusses for which questions and in which fields TA can offer such assistance, by discussing several studies of the Austrian Institute for Technology Assessment.
\end{abstract}

\title{
Inhalt
}

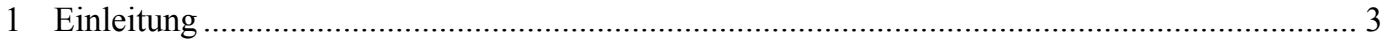

2 Fragen, die TA nicht beantworten kann (und darf).............................................................. 4

3 Biotechnologie ist dennoch ein Arbeitsschwerpunkt von TA............................................... 7

4 Beispiele für TA-Studien des ITA................................................................................. 10

4.1 E-democracy: Ausleuchtung des Potentials einer spezifischen Technologie-Anwendung ................ 10

4.2 Das digitale Krankenhaus: Integration einer neuen Technologie in ein bestehendes System........... 11

4.3 HTA: Screening vielfach interessengeleiteter Studien ................................................................. 12

4.4 Cyberscience: Folgen der Digitalisierung für die Wissenschaft ................................................. 14

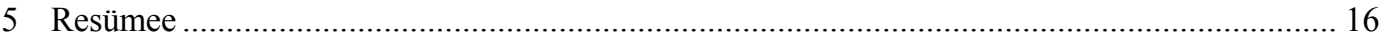

Anhang A: Das Institut für Technikfolgen-Abschätzung der ÖAW ........................................... 17

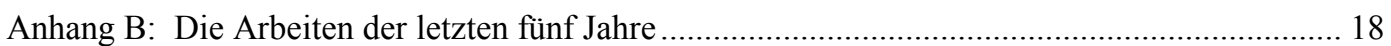

Rede gehalten vor dem Verein der Freunde der Österreichischen Akademie der Wissenschaften am 20. April 2004. 


\section{Einleitung}

Er kenne keine Technik, die durch Technikfolgen-Abschätzung ermöglicht oder verhindert worden wäre, meinte jüngst ein Mitglied dieser Akademie. Diese Beobachtung ist nicht unrichtig, verkennt aber die Aufgaben von Technikfolgen-Abschätzung (TA). Technikfolgen-Abschätzung wurde notwendig, weil unsere Welt - Wirtschaft wie Gesellschaft - nicht zuletzt durch die Kumulierung von Wissen zunehmend komplexer geworden ist. Zunehmende Spezialisierung war die unvermeidliche Reaktion; sie ermöglichte zwar den beeindruckenden wissenschaftlichen Fortschritt der letzten Jahrzehnte, erschwert aber gesamthafte Beurteilungen, die zwangsläufig breites und fachübergreifendes Wissen erfordern. TA versucht diese Lücke im Wege interdisziplinärer Zusammenarbeit zu füllen und Entscheidungsunterlagen überall dort aufzubereiten, wo sich ein und dasselbe Problem aus dem Blickwinkel unterschiedlich spezialisierter Experten unterschiedlich darstellt, demgemäß zu unterschiedlichen Expertisen und damit auch zu unterschiedlichen, vielfach sogar einander widersprechenden Entscheidungsgrundlagen führt.

Ich werde zunächst herausarbeiten, zu welchen Fragen und auf welchen Gebieten TA solche Entscheidungshilfen überhaupt bieten kann, und in weiterer Folge die Möglichkeiten von TA an Hand einiger Arbeiten des ITA, des Instituts für Technikfolgen-Abschätzung der Österreichischen Akademie der Wissenschaften, aufzeigen. Zuerst jedoch, zur Veranschaulichung, ein Beispiel aus unserer Arbeit, das die unterschiedlichen Perspektiven unterschiedlich spezialisierter Experten illustriert: Es geht dabei um die Beurteilung der Grünen Bioraffinerie, einer Anlage, die aus grüner Biomasse wie Gras, Getreide, u.ä. verschiedene Produkte wie Milchsäure, Proteine und Biogas erzeugen soll. Als Technologie, die nachwachsende Rohstoffe verarbeitet, wird sie als ,nachhaltig“ angesehen. ITA hat in seinem Projekt TA der Grünen Bioraffinerie ${ }^{1}$ die unterschiedlichen Expertisen zur Bewertung dieser Technologie zusammengestellt und ist dabei auf sehr differenzierte Sichtweisen gestoßen. So sehen etwa Agrarexperten das Projekt primär unter dem Aspekt der Verwertung von Agrarüberschüssen, Planungsingenieure unter dem der technischen Machbarkeit, Betriebwirte unter dem der Rentabilität, Umweltexperten unter dem der ökologischen Auswirkungen. Unvermeidlicherweise entstehen dabei Zielkonflikte; ein markantes Beispiel: Die Techniker optimieren die Verfahren, woraus sich Art und Menge des Output bestimmen; die Betriebswirte schätzen an Hand der erzielbaren Marktpreise und der erforderlichen Kapitalverzinsung die höchstmöglichen Inputpreise ab; die Agrartechniker stellen fest, dass solche Preise bloß durch erhebliche Intensivierung und Technisierung erzielt werden könnten; die Bauernvertreter setzen dem entgegen, dass es nicht um Intensivierung sondern um Überschussverwertung und Einkommenssicherung lokaler Produzenten gehe, und für die Ökologen sind Intensivierung und Umweltschutz unvereinbar. Jeder hat aus seiner Sicht Recht, aber die Nachhaltigkeit, der Auslöser des Projekts, ist verloren gegangen; es droht eine Lösung, die niemanden befriedigt, die keines der Ziele erreicht, aber Fördermittel verbraucht. TA kann in seiner Mediationsfunktion versuchen, eine gemeinsame Diskussionsbasis für die Experten zu schaffen. ITA hat in diesem Fall einen Kriterienkatalog zur Operationalisierung von Nachhaltigkeit entwickelt, potentielle Zielkonflikte anhand dieser Kriterien ausgearbeitet und Handlungsoptionen zur Unterstützung künftiger Entscheidungen zur Weiterentwicklung bzw. Förderung der Grünen Bioraffinerie im Sinne einer nachhaltigen Entwicklung abgeleitet. Interessant ist, dass weder die Betreiber des Projekts, die Interessenvertretungen, noch die Entscheidungsbefugten, die Ministerien, den Forschungsbedarf erkannten: Jeder hielt die Argumente der jeweils eigenen spezialisierten Experten für die einzig richtigen, die der Anderen für interessengeleitet. ${ }^{2}$

1 Schidler, S., 2003, Technikfolgenabschätzung der Grünen Bioraffinerie, Teil I: Endbericht, Institut für Technikfolgen-Abschätzung, Juli, http://www.oeaw.ac.at/ita/ebene5/d2-2d2I.pdf.

2 ITA musste demgemäß dieses Projekt aus Eigenmitteln finanzieren. 
Ein Streit der Gefälligkeitsgutachten? Keineswegs! Das kleine Beispiel aus der täglichen Praxis zeigt vielmehr ein wichtiges Problem bei der Zusammenarbeit spezialisierter Experten: Spezialisten haben sich nicht bloß als Folge der Komplexität unserer Welt und aus Effizienzgründen auf ein Gebiet spezialisiert; sie haben es nicht zuletzt deswegen getan, weil sie ihr Spezialgebiet für wichtig halten, für wichtiger als andere Fachgebiete; sie lieben ihr Fachgebiet und sie kämpfen für ihre Erkenntnisse. Für das Spitzenwissen auf ihrem Spezialgebiet müssen sie jedoch durch Minderkenntnis auf anderen Gebieten büßen. Es bedarf daher mediatisierender Generalisten, um die spezialisierten Experten auf die Erkenntnisse der Spezialisten anderer Fachgebiete und auf die Relevanz auch anderer Ziele zu verweisen. TA kann das umso eher leisten, je unterschiedlicher, je breiter gestreut die beteiligten Fachgebiete sind.

Mediatisierungs-Institutionen ${ }^{3}$ können darüber hinaus auch einen wichtigen Beitrag zur öffentlichen Debatte über die Rolle der Wissenschaft bzw. des Experten in der modernen Gesellschaft leisten. Sie können der communis opinio entgegenwirken, derzufolge zwei Experten grundsätzlich drei Meinungen haben, und jedes Argument wie auch sein Gegenteil von Experten jederzeit gutachterlich begründet werden kann; ${ }^{4}$ sie können mithelfen, das Bild und die Bedeutung der Wissenschaft in der Öffentlichkeit zu verbessern, in dem sie klar machen, dass unterschiedliche Expertenmeinungen entweder aus der Konzentration auf unterschiedliche Einzelaspekte resultieren - wie das am Beispiel der Grünen Bioraffinerie gerade gezeigt wurde -, oder aus neuen Fragestellungen, die wissenschaftlich noch nicht geklärt sind, und bei denen es daher noch keine einheitliche Meinung geben kann. Ich komme auf diesen Punkt noch zurück.

\section{Fragen, die TA nicht beantworten kann (und darf)}

Nun aber zu der wichtigen Frage, auf welchen Gebieten TA ihre Mediatisierungsfunktion bzw. ihren Beitrag zur Entscheidungsfindung überhaupt leisten kann. Konkreter und schärfer formuliert: Wo sind die TA-Studien, die die für TA reklamierte Mediatisierung bei dem Streit um die Atomtechnik, um die Grüne Gentechnik, um das Klonen versuchen; wo sind die TA-Studien, die Entscheidungsgrundlagen für die Förderung oder Verhinderung dieser Techniken liefern, wie das der einleitend zitierte Kollege von TA verlangte? Wo ist der TA-Experte, den Senator J.O. Pastore im Hearing on „The Status of Nuclear Safety” im US Congress Joint Committee on Atomic Energy (Washington, DC 1973) suchte: „I wish just once some scientist would come here and tell us whether or not nuclear power is or isn't safe."? Dass es diesen Experten nicht geben kann, ist klar - solange wir Scharlatane nicht unter die Experten zählen; doch auch die Mediatisierungsversuche hielten sich auf diesen Gebieten in Grenzen, jedenfalls was ihren Erfolg betrifft. Sie halten sich allerdings nicht deswegen in Grenzen, weil die TA-community etwa vor diesen Fragen zurückschreckt; zahlreiche Studien haben klar herausgearbeitet, warum diesbezügliche Mediatisierungsversuche scheitern müssen. Vier Argumente spielen dabei eine besondere Rolle:

3 Der Begriff Mediatisierungs-Institutionen wird hier breit verstanden: Es geht keineswegs bloß um Wissenschafter und deren Arbeit, sondern um die unterschiedlichen Einrichtungen partizipativer Art.

4 Siehe dazu Tichy, G., 2002, Der Volkswirt als Politikberater, in G. Chaloupek et al, Hg, Ökonomie in Theorie und Praxis, Festschrift für Helmut Frisch, Berlin: Springer, 359-77. 
- Erstens eignen sich JA/NEIN-Fragen generell nicht für Mediatisierung im Allgemeinen und schon gar nicht für TA. Denn nach der Definition des Vereins Deutscher Ingenieure ${ }^{5}$ ist Technikfolgen-Abschätzung

„das planmäßige, systematische, organisierte Vorgehen, das den Stand einer Technik und ihre Entwicklungsmöglichkeiten analysiert, unmittelbare und mittelbare technische, wirtschaftliche, gesundheitliche, ökologische, humane, soziale und andere Folgen dieser Technik und möglicher Alternativen abschätzt, aufgrund definierter Ziele und Werte diese Folgen beurteilt oder auch weitere wünschenswerte Entwicklungen fordert, Handlungs- und Gestaltungsmöglichkeiten daraus herleitet und ausarbeitet, so dass begründete Entscheidungen ermöglicht und gegebenenfalls durch geeignete Institutionen getroffen und verwirklicht werden können.“

TA kann, soll und muss daher die unmittelbaren und mittelbaren technischen, wirtschaftlichen, gesundheitlichen, ökologischen, humanen, sozialen und anderen Folgen unterschiedlicher Anwendungen von Gentechnik oder Atomtechnik als solche herausarbeiten; sie kann und darf aber nicht versuchen, etwa die wirtschaftlichen und gesundheitlichen Folgen gegeneinander aufzurechnen - das ist eine genuin politische Entscheidung, die allein der Souverän oder die von ihm gewählten und ihm verantwortlichen Politiker treffen können. Und genau so unzulässig ist es, die - eventuell unterschiedlichen - Bewertungen von Gentechnik in den Bereichen Pflanzenzüchtung, Tierzüchtung, Medizin oder Klonen zu einer Bewertung der Gentechnik als solcher zu aggregieren.

- Zweitens gibt es einen Konflikt der Risikokonzepte: Die öffentliche Diskussion und die Sozialwissenschafter gehen von einem Konzept des Gesellschaftlichen Risikos aus, das alles umfasst, was durch die öffentliche Diskussion, ,aus der unbegrenzten Fülle von Handlungen, die mit Ungewissheit und möglichen Schäden verknüpft sein können - also aus dem Schattenreich der Gefahr - herausgeholt wurde, dass es durch gesellschaftliche Diskurse thematisiert und benennbar wurde, abgrenzbar und letztlich abwägbar. ... Gefahren werden gleichsam zivilisatorisch zugewiesen."6 Das heißt nichts anderes, als dass die gesellschaftliche Diskussion letztlich bestimmt, wovor sich die Leute fürchten, was sie akzeptieren und was sie ablehnen. Experten aus den Bereichen Naturwissenschaften und Technik können mit einem solchen, wenig objektiven und für sie unklaren Konzept zumeist wenig anfangen; sie tendieren eher zu einem technisch orientierten Risikobegriff mit starker Betonung von Eintrittswahrscheinlichkeit und Schadenshöhe; dieser leidet allerdings darunter, dass für seltene Ereignisse Eintrittswahrscheinlichkeiten nicht abgeschätzt und die eventuell abschätzbaren Einzelrisiken nicht zum Systemrisiko aggregiert werden können. ${ }^{7}$ Auch ist der technisch orientierte Risikobegriff nicht das Konzept, nach dem die Entscheidungen in demokratischen Institutionen fallen (können).

- Drittens geht es, wie van den Daele und Bora herausgearbeitet haben, bei dem Streit um Gefahren und Risiken von Atom- oder Gentechnik gar nicht um Sachfragen oder um einen Konflikt unterschiedlicher Risikokonzepte, sondern um Ideologien, um einen Streit zwischen konkurrierenden Weltbildern: V.d. Daele ${ }^{8}$ konfrontierte 1991/93 in einer Reihe von Konferenzen etwa 60 Befürworter und Gegner in einem ,herrschaftsfreien Diskurs' à la Habermas und sorgte dafür, dass strittige Themen stets ausdiskutiert wurden. Der Versuch einer Konsensfindung scheiterte: Formal, weil die Gentechnik-Gegner den Diskurs abbrachen, materiell, weil sich herausstellte, dass hinter den vorgebrachten Argumenten grundsätzlich unterschiedliche weltanschaulich-moralische Motivationen bzw. Utopien standen, und, daraus resultierend, unterschiedliche Einstel-

5 VDI 1981

6 Evers, A. und H. Nowotny, 1987, Über den Umgang mit Unsicherheit, Frankfurt/M.: Suhrkamp, 34ff.

7 Siehe dazu Tichy, G., 2003, Die Risikogesellschaft - Ein vernachlässigtes Konzept in der europäischen Stagnationsdiskussion, ITA manu:script, ITA-03-02, http://www.oeaw.ac.at/ita/pdf/ita_03_02.pdf.

8 Enquete-Kommission des Deutschen Bundestages, 1987, Chancen und Risiken der Gentechnologie, München: Schweitzer (Bundestagsdrucksache 10/6775). 
lungen zur Technik. ${ }^{9}$ In dieselbe Richtung einer ,sachliche[n] Unabschließbarkeit von Risikokontroversen" weist auch die Untersuchung der Diskussionen anlässlich der Erörterungstermine zur Freisetzung genetisch veränderter Pflanzen in Deutschland: ${ }^{10}$ Man redete aneinander vorbei, die Konflikte wurden nicht überwunden sondern sogar verhärtet und politisiert. ${ }^{11}$

- Viertens haben Studien der Technikfolgen-Abschätzung die Hoffnung der meisten Wissenschafter erschüttert, die Ablehnung wissenschaftlicher oder technischer Optionen durch die Öffentlichkeit mittels besserer Aufklärung, mittels Bemühungen um 'public understanding of science' überwinden zu können. Das EU-Projekt Zur öffentlichen Wahrnehmung der Biotechnologie, an dem ITA maßgeblich mitwirkte, konnte herausarbeiten, dass der vermeintlich direkte Zusammenhang zwischen Faktenwissen und Akzeptanz ein Mythos ist. Viel stärker als Wissen bestimmen Nutzenerwägungen oder moralische Bewertungen die Akzeptanz oder Ablehnung einer bestimmten Technik. Viel Energie und Geld wird verschwendet, weil dieser gut abgesicherten Erkenntnis nicht gefolgt wird.

Der vielfach erhoffte aber leider nicht existierende Zusammenhang von Wissen und Akzeptanz, dessen Fehlen vielfach unter dem verengenden Schlagwort, Technik- und Wissenschaftsfeindlichkeit' abgehandelt wird, hat für die Wissenschaftspolitik generell und die ÖAW speziell besondere Bedeutung - über seinen spezifischen TA-Aspekt hinaus: Bemüht sich die ÖAW doch mit Symposien $^{12}$ wie mit der Errichtung der „Galerie der Forschung“ in der Alten Aula der Jesuitenuniversität, die breite Öffentlichkeit und die Medien von der Bedeutung der Wissenschaft zu überzeugen und deren Akzeptanz zu verbessern. Für diese Bemühungen ist das Ergebnis der ,public understanding of science'-Debatte von zentraler Bedeutung: dass nämlich die Vermittlung von Wissen als solchem zu Verständnis und Akzeptanz wenig beiträgt, dass sich Einstellungen auf diesem Weg kaum ändern lassen. Will man das Bild der Wissenschaft in der Öffentlichkeit verbessern, wird man um den mühsamen Weg nicht herumkommen, Wissenschaft als dialektischen Prozess des Aufstellens und Falsifizierens von Thesen begreiflich zu machen. Es gilt zu vermitteln, dass sich die Wissenschaft ausschließlich mit noch ungelösten und daher zwangsläufig neuen und demgemäß strittigen Fragen beschäftigt, ${ }^{13}$ sodass Wissenschafter unvermeidlicherweise unterschiedliche Meinungen haben müssen; denn allein durch die Arbeit an unterschiedlichen Ansätzen, durch Aufstellen und Falsifizierung unterschiedlicher Thesen ist wissenschaftliche Erkenntnis möglich: als asymptotische Annäherung an die ,Wahrheit'.

9 Enquetekommission, 44.

10 Bora, A., 1999, Differenzierung und Inklusion. Partizipative Öffentlichkeit im Rechtssystem moderner Gesellschaften, Baden-Baden:

11 „Für das Weltbild der modernen Wissenschaft wirft die „Veränderung der Natur durch den Menschen ... keine moralischen Fragen auf. ... Im Weltbild der bürgerlichen Gesellschaft wird die Person zum alleinigen Träger der Wert- und Sinnhaftigkeit der Welt. Autonomie wird der höchste Wert, und inhaltliche moralische Bindungen des Verhaltens werden tendenziell durch Freiheiten ersetzt, ... Die gegenläufige Tendenz der ,Fundamentalisierung' stellt dem Ressourcenkonzept von Natur einen remoralisierten Naturbegriff gegenüber, erneuert gegenüber der Moral der Freiheit, absolute gleichsam mit Tabucharakter versehene moralische Forderungen ..." (W. v.d. Daele, Risiko-Kommunikation: Gentechnologie, in H. Jungerman et al, Hg, 1991, Risikokontroversen. Konzepte, Konflikte, Kommunikation, Berlin: Springer, 15). In demselben Sinn Jonas (1984, 57): „Denn ebendieselbe Bewegung, die uns in den Besitz jener Kräfte gesetzt hat, deren Gebrauch jetzt durch Normen geregelt werden muss - die Bewegung des modernen Wissens in Gestalt der Naturwissenschaft - hat durch eine zwangsläufige Komplementarität die Grundlagen fortgespült, von denen Normen abgeleitet werden konnten, und hat die bloße Idee von der Norm als solcher zerstört.“

12 Communicating Science: Concepts, Programs, Experiences, 18.-20. September 2003 in der ÖAW.

13 Allein (noch) strittige Fragen sind Gegenstand wissenschaftlicher Beschäftigung; unstrittige, also gelöste Probleme fallen der Trivialisierung anheim und werden vielfach zu Elementen der Allgemeinbildung: $\mathrm{Zu}$ Zeiten Galileis etwa war die Frage (noch) strittig, ob die Sonne um die Erde kreist oder umgekehrt - es war eine wissenschaftliche Streitfrage; heute ist diese Frage geklärt und damit nicht mehr ein Thema der Wissenschaft; diese hat sich neuen, ungelösten und daher strittigen Fragen zugewandt. 


\section{Biotechnologie ist dennoch ein Arbeitsschwerpunkt von TA}

Die bisherigen Ausführungen sollten klar machen, dass eine intensive Beschäftigung der Technikfolgen-Abschätzung mit Atom- bzw. Gentechnik als solcher selbst dann wenig hilfreich wäre, wenn es nicht um ,Ja oder Nein' sondern bloß um die Aufbereitung von Entscheidungsgrundlagen ginge: Mediatisierung, Vermittlung zwischen den unterschiedlichen Meinungen ist in diesen Fällen aussichtslos, Aufbereitung von Entscheidungsgrundlagen zwecklos; die Akteure haben ihre festgefügten Vorstellungen, von denen sie sich nicht abbringen lassen, sie haben keinen Bedarf an zusätzlicher Information.

Die Erkenntnis, dass Atomtechnik oder Gentechnik als solche nicht Gegenstand der Technikfolgen-Abschätzung sind, schließt jedoch nicht aus, dass diese Themenfelder von TA im Allgemeinen und vom ITA im Besonderen bearbeitet werden; es geht dabei allerdings entweder um Grundlagen oder um spezifische Aspekte, nicht um die jeweilige Politik als solche. Aus der Arbeit des ITA habe ich das EU-Projekt Zur öffentlichen Wahrnehmung der Biotechnologie (LSES), ${ }^{14}$ schon erwähnt, das sich mit der Wahrnehmung der Gentechnik in der Presseberichterstattung und den daraus resultierenden Folgen für die Politik beschäftigt. Das Projekt ist deswegen so besonders wichtig, weil eine Implementationsstrategie, die die Chancen der Gentechnik zu wahren sucht, die Besorgnisse wichtiger Gruppen und die öffentliche Kritik nicht außer Acht lassen kann; sie scheiterte sonst an ihrer Durchsetzbarkeit. Doch: ist das nicht alles längst bekannt? Weiß man nicht längst, dass es der Öffentlichkeit an Fachwissen mangelt, dass sie von den Vorzügen eben erst überzeugt werden muss, dass sie generell technikfeindlich ist, und gerade in Bezug auf Gentechnik von den Medien und den NGOs aufgehetzt wird? Das LSES-Projekt enthüllte die meisten dieser Behauptungen als Mythen, die einer wissenschaftlichen Überprüfung nicht standhalten.

Den Mythos der Akzeptanzbeschaffung durch Wissensvermittlung habe ich schon behandelt, der Versuch ist ebenso teuer wie unwirksam. Doch auch die behauptete Technikfeindlichkeit der europäischen Öffentlichkeit enthält erhebliche Elemente eines Mythos: Zwar reagieren Europäer auf

14 G. Gaskell et al, 2003, Europeans and Biotechnology in 2002, Eurobarometer 58.0, A report to the EC Directorate General for Research from the project 'Life Sciences in European Society' QLG7-CT-1999$00286,2^{\text {nd }}$ ed., europa.eu.int/comm/public_opinion/archives/eb/ebs_177_en.pdf, sowie die Vorgängerprojekte Biotechnology in the European Public Concerted Action (BEP, 1996-98), und European Debates on Biotechnology Concerted Action (EUDEB, 1998-2000). Daraus resultierten Publikationen mit ITA-Mitarbeitern als Autoren und Koautoren: Biotechnology and the European Public Concerted Action group, 1997, Europe Ambivalent on Biotechnology, NATURE 387, pp. 345-347; Gaskell, G.et al, 2000, Biotechnology and the European public, Nature Biotechnology 18(9), pp 935-938; Wagner, W. et al, 1998, Austria (country report), in J. Durant et al eds, Biotechnology in the Public Sphere: a European Sourcebook, London: Science Museum Press, pp. 15-28; Torgersen, H. et al, 2002, Promise, problems and proxies: twenty-five years of debate and regulation in Europe, in Bauer, M. and G. Gaskell eds, Biotechnology. The Making of a Global Controversy, Cambridge University Press, pp. 21-94; Einsiedel, E. et al, 2002, Brave new sheep - the clone named Doly, ibid., pp. 313-347; Grabner, P. et al, 2001, Bio-Political Diversity: The Challenge of Multi-Level Policy-Making, in Gaskell, G. and M. Bauer eds, Biotechnology 1996-2000. The years of controversy, London: Science Museum Press, pp 15-34,; Gaskell, G. et al, 2001, Troubled Waters - The Atlantic Divide on Biotechnology Policy, ibid, pp. 96-115; Gaskell, G. et al., 2001, Biotechnology, Technoscience and the Public Sphere, ibid, pp.116-27; Torgersen, H. et al, 2001, Austria: Narrowing the Gap to Europe, ibid, pp. 131-44; Grabner. P. and H. Torgersen, 1998, Österreichs Gentechnikpolitik Technikkritische Vorreiterrolle oder Modernisierungsverweigerung? Österreichische Zeitschrift für Politikwissenschaft 1/98, 5-27; Torgersen, H. et al, 2000, Europe the spoil-sport. On the Europeans' reluctance towards genetically modified food, J. of Biolaw and Business 3(3), pp. 53-60; Torgersen, H., 2002, Austria and the Transatlantic Agricultural Biotechnology Divide, Science Communications 2(2), pp.173183. 
neue Technologien weniger enthusiastisch als etwa Amerikaner, doch eine generelle Technikfeindlichkeit lässt sich nicht belegen. ${ }^{15}$ In der Gentechnik bewertet die europäische Öffentlichkeit vor allem medizinische Anwendungen durchwegs und sogar in zunehmendem Ausmaß positiv. Die grüne Gentechnik hingegen genießt keinen guten Ruf, vor allem wenn es um Nahrungsmittel geht; sie wird aber in den meisten Ländern zumindest nicht dezidiert abgelehnt. Und dort wo der Widerstand groß ist, resultiert er weniger aus Risikoerwägungen als vielmehr aus kulturellen Leitbildern betreffend Natur und Landwirtschaft, aus Leitbildern, die im Zuge von Regulierungsentscheidungen in Form von Risikoargumenten politisch virulent werden.

Auch der dominierende Einfluss der Medien und der NGOs erwies sich in der LSES-Studie als Mythos, ein Mythos allerdings, der von beiden Gruppen sorgsam gepflegt wird, weil er die Basis ihrer Macht bildet: Die europäische Öffentlichkeit war längst Gentechnik-skeptisch, als die NGOs das Thema entdeckten, die schon vorhandene Ablehnung organisierten und medienwirksam verstärkten. Auch die negativen Kampagnen von Boulevardblättern konnten bloß eine bereits vorhandene öffentliche Meinung verstärken (nicht jedoch selbst auslösen); um wirksam zu werden, benötigen diese Kampagnen überdies Auslöseschocks (Trigger-Ereignisse), die die Öffentlichkeit beunruhigen; auch waren sie i. d. R. zeitlich bloß begrenzt wirksam. Qualitätszeitungen hingegen, die nachgewiesenermaßen die Meinungsführerschaft haben, äußerten sich in der Summe eher positiv über Gentechnik. Nun mag es für Anhänger der Gentechnik eine unerfreuliche Botschaft sein, dass nicht Unwissen, Aktionismus der NGOs oder Verhetzung durch den Boulevard hinter der zurückhaltenden Beurteilung der Öffentlichkeit stehen, sondern kulturelle Leitbilder und Nutzen-/Risikoüberlegungen der Bürger; aber es ist selbst für sie eine wichtige Botschaft, denn sie hilft ihnen, sich nicht im sinnlosen Kampf gegen Mythen zu verzetteln und sich den wahren Ursachen zuzuwenden.

Das LSES-Projekt, das vor kurzem abgeschlossen wurde, ließ erkennen, dass bei der Beurteilung der Gentechnik vielfach - z. T. sogar unbewusst - kulturelle Leitbilder maßgebend sind, zum Beispiel und gerade auf dem Teilgebiet der Humangenetik. Das ist von nicht zu unterschätzender Bedeutung für die politische Implementation, Regulierung und/oder Steuerung. Das weiterführende Projekt Kulturelle Leitbilder in der Humangenetik dient der Vertiefung der Erkenntnis auf diesem Gebiet. Abermals geht es um die Grundlagen für politische Implementation, Regulierung und/oder Steuerung; das mag esoterisch klingen, ist aber von zentraler Bedeutung. Ohne Wissen um die einer Technologie zugrunde liegenden kulturellen Leitbilder bleiben Implementations- oder Steuerungsversuche oberflächlich und i. d. R. zum Scheitern verurteilt: Technik lässt sich nicht dekretieren. Der andauernde Konflikt um Nutzen und Gefahren der Biotechnologie in der Landwirtschaft ist ein aktuelles Beispiel. Deutlicher vielleicht lässt sich die Relevanz von Leitbildern am alltagsnahen Beispiel des Autos demonstrieren. Das System ,Auto' ,funktioniert' nur auf der Basis bestimmter Mobilitäts- und Statusvorstellungen der Gesellschaft: Ohne Mobilitätswahn kein Fetisch PKW. Kennt man die Mobilitätsvorstellungen nicht, sind die schönsten Verkehrskonzepte sinnlos - das Auto fährt der Politik immer davon, und leider ist es genau das, was wir auch tatsächlich beobachten. In ähnlicher Weise setzt auch die Humangenetik allgemein geteilte Normalitätsvorstellungen voraus, ,funktioniert' also nur unter dieser Voraussetzung. Die kulturellen Leitbilder, die Vorstellungen darüber, was gesund und krank ist, was ein lebenswürdiges Leben, was bereits Leben ist und was noch nicht, finden sich in den Alltags- und Expertendiskursen zu spezifischen Leitbildern verdichtet: Das Konzept der ,Person', an dem sich das Handeln und Entscheiden der Experten orientiert, das Konzept des ,mündigen Patienten' bzw. der Patientenautonomie sind solche Leitbilder, die die Regulierung der genetischen Beratung bestimmen. Eine Entscheidung des Humangenetikers über den Kopf des Klienten hinweg wäre in unseren Tagen undenkbar, sie würde Erinnerungen an

15 In Europa kann man nicht von einer einheitlichen Entwicklung sprechen: Der Grad an Akzeptanz (oder Nichtakzeptanz) hat sich in manchen Ländern (wie etwa Österreich) relativ wenig verändert, in anderen (insbesondere solchen, die anfangs - bei besonders geringem Wissen - eher positiv eingestellt waren) wie Griechenland, aber auch bei Frankreich ergab sich ein dramatischer Umschwung. 
das Zeitalter der Eugenik wecken. Jede Technologie repräsentiert eben eine bestimmte Kultur, sie ist ein Stück ,gehärteter' Normalität, „Technology is society made durable” (Bruno Latour). TAForschung zu kulturellen Leitbildern ist ein wichtiger Bestandteil reflektierter Politikberatung, und von zentraler Bedeutung im Kontext der Überarbeitung des österreichischen Gentechnikgesetzes, das auch die Anwendung humangenetischer Diagnostik und Beratung regelt.

Ein letztes, eher technisches Beispiel aus dem Bereich der Biotechnologie sei angeführt, um nicht den Eindruck zu erwecken, wir würden uns ausschließlich mit der Bewusstseinsbildung als Grundlage von Regulierungsentscheidungen befassen. In drei aufeinander folgenden internationalen von der EU bzw. nationalen Stellen geförderten Projekten haben wir uns mit der Regulierung gentechnisch veränderter Nutzpflanzen und der Entwicklung konsistenter Kriterien für die Risikoabschätzung beschäftigt. ${ }^{16}$

Hierbei ergibt sich das Dilemma, dass eine EU-weite Harmonisierung einheitliche Kriterien voraussetzt, die lokalen Gegebenheiten - Klima, Vegetation oder auch die landwirtschaftliche Struktur - sich jedoch in den einzelnen Ländern stark unterscheiden; demgemäß unterschiedlich sind die Anforderungen an die Abschätzung. So ist etwa die Wahrscheinlichkeit der Übertragung eines eingeführten Gens auf andere Pflanzen nicht nur vom Vorhandensein geeigneter Empfängerpflanzen abhängig, sondern auch von der der Größe der Felder; die Kleinräumigkeit, etwa der österreichischen Landwirtschaft, spielt hier eine nicht unbedeutende Rolle. Darüber hinaus sind es wieder die unterschiedlichen kulturellen Leitbilder, die Schwierigkeiten verursachen: was etwa unter einer ,natürlichen Umwelt' zu verstehen ist, die es zu schützen gilt, wird in den Niederlanden anders gesehen als etwa in Österreich. Die unterschiedliche Einschätzung der „Grünen Gentechnik“ in einzelnen Ländern ist ohne diesen Hintergrund nicht zu verstehen. Gemeinsam mit der Forderung nach größtmöglicher Harmonisierung sind daraus demokratiepolitische Probleme und eine Lähmung der Politik auf EU-Ebene entstanden, die zu einem zeitweiligen Moratorium für Anträge auf Markteinführung gentechnisch veränderter Produkte geführt haben. Österreich scheint hierbei frühzeitig ein Beispiel geliefert zu haben, dass aus vermeintlichen technischen Sachzwängen resultierende Strategien an den geschilderten Widrigkeiten scheitern können.

Einen wesentlichen Beitrag zu einer verbesserten Entscheidungsfindung versprachen sich viele von der konsequenten Umsetzung des Vorsorgeprinzips in diesem Bereich. Eine europaweite empirische Untersuchung, an der das ITA mitwirkte, ${ }^{17}$ zeigte allerdings, dass maßgebliche Akteure stark divergierende Auffassungen über die praktische Bedeutung und die angemessene Umsetzung des Vorsorgeprinzips haben. Dahinter stehen divergierende Vorstellungen über das Verhältnis von Wissenschaft und Politik, die - anders als die EU-Kommission mit ihrer Mitteilung zum Vorsorgeprinzip meinte - per Dekret nicht zu beseitigen sind. Unsere Studie zeigte, dass es nicht genügt, einzig das Vorsorgeprinzip zu bemühen; eine an Vorsorge orientierte Politik muss vielmehr die unterschiedlichen und vielfach gegensätzlichen Anforderungen in den einzelnen Ländern berücksichtigen.

16 EU-Kommission DG Research, Precautionary Expertise of GM Crops (PEG), und die Vorgängerprojekte: GMO Releases: Managing Uncertainties About Biosafety (1995-1996), Safety Regulation of Transgenic Crops (1997-1999). Daraus resultierende Publikationen mit ITA-Mitarbeitern als Autor oder Koautor: Levidow, L. and S. Carr eds, 1996, Biotechnology Risk Regulation in Europe, Science and Public Policy, special issue, 23; Levidow, L. et al eds, 2000, Genetically modified crops in the European Union, J. Risk Research, special issue 3; Grabner, P. and H. Torgersen, Österreichs Gentechnikpolitik - Technikkritische Vorreiterrolle oder Modernisierungsverweigerung? Österreichische Zeitschrift für Politikwissenschaft 1/98, 5-27; Mikl, M. and H. Torgersen, 1996, Austria's biotechnology regulation: from 'virtual releases' to public protest, Science and Public Policy 23(3), pp. 195-200; Torgersen, H. and F. Seifert, 2000, Precautionary Blockage of Agricultural Biotechnology, J. Risk Research 3(3), pp. 209-217.

17 Scientific Basis of Applying the Precautionary Principle, im Auftrag des European Science and Technology Observatory network (ESTO) der EU-Kommission. Daraus resultierende Publikationen mit ITA-Mitarbeitern als Autor oder Koautor: Torgersen, H. and F. Seifert, 1997, Aversion Preceding Rejection: results of the Eurobarometer survey 39.1 on biotechnology and genetic engineering in Austria, Public Understanding of Science 6/97, 1-12; Seifert, F. and H. Torgersen, 1997, How to keep out what we don't want. An as- 


\section{Beispiele für TA-Studien des ITA}

Die drei Beispiele aus der Biotechnologie lassen schon die Forschungsrichtung und die Absichten - Neudeutsch die ,Mission' - von TA im Allgemeinen und von ITA im Besonderen erkennen: Analyse neuer Technologien und Herausarbeitung ihrer unterschiedlichen Folgen, um daraus Handlungs- wie Gestaltungsmöglichkeiten für die Politik, aber auch für Gestalter und Anwender abzuleiten. Vier Beispiele aus den anderen Arbeitsgebieten des ITA sollen die unterschiedlichen, durch die jeweilige Problemstellung bedingten Stossrichtungen von TA zeigen. Im ersten Beispiel geht es um die Ausleuchtung des Potentials einer spezifischen Technologie-Anwendung, im zweiten um die Integration einer neuen Technologie in ein bestehendes System, im dritten um ein Screening vielfach interessengeleiteter Studien, und im vierten um die Folgen der Digitalisierung für einen riesigen, stark differenzierten Gesamtkomplex, nämlich die Wissenschaft.

\section{I E-democracy: Ausleuchtung des Potentials einer spezifischen Technologie-Anwendung}

Bei E-democracy, ,elektronischer Demokratie' geht es um politische Partizipation mittels neuer Informations- und Kommunikationstechnologien. Dahinter steht die Sorge um rückläufige Wahlbeteiligung, ${ }^{18}$ Verlust des Vertrauens von BürgerInnen in rechtsstaatliche Einrichtungen und ihre RepräsentantInnen, die gemeinsam mit reduzierter Informations- und Meinungsvielfalt am Medien-

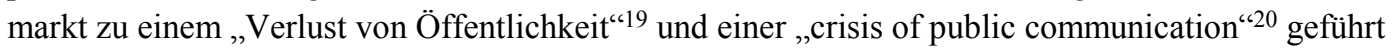
haben. Diese fortschreitende Entfremdung erscheint vor dem Hintergrund der Erweiterung und Vertiefung der Europäischen Union besonders problematisch. Die EU versucht dem u. a. mittels webbasierter Dienste entgegenzuwirken. BürgerInnen, Unternehmen und NGOs werden aufgefordert, in Online-Debatten und Online-Konsultationen zu europäischen Themen Stellung zu beziehen. Wieweit dieser Versuch hilfreich ist, untersuchen wir in dem vom Bildungsministerium geförderten Projekt Europeans have a say: Online debates and consultations in the European Union: Wir durchleuchten die bisherigen Beiträge im Hinblick darauf, inwieweit wirklich ,deliberative' Diskurse stattfinden, d. h. Kommunikationsprozesse mit einem hohen Grad an Interaktivität und einem qualitativ hohen Diskursniveau. ${ }^{21}$

sessment of 'Sozialverträglichkeit' under the Austrian Genetic Engineering Act. Public Understanding of Science, 6/97, pp. 301-27; Torgersen, H., 2001, Precautionary openness. Understandings of precaution as an indicator for change in biotechnology policy, Politeia XVII(63), pp 67-79; Millstone E. et al., forthcoming, Science and Governance in Trade-Related Risk Regulatory Decision-Making: Comparative Case Studies, Report to ESTO.

18 In Österreich sank die Wahlbeteiligung bei Nationalratswahlen von 94 \% im Jahre 1945 auf $84 \%$ in 2002 (BM f. Inneres), in den USA ist sie (infolge von Brüchen ohne Daten für die Südstaaten) von $85 \%$ zu Beginn des vorigen Jahrhunderts auf rund $70 \%$ in den fünfziger Jahren und auf zuletzt knapp über $50 \%$ gefallen (R.D. Putnam, 2001, Bowling alone, New York: Simon \& Schuster, 32).

19 Habermas, J., 1962, Strukturwandel der Öffentlichkeit. Untersuchungen zu einer Kategorie der bürgerlichen Gesellschaft, Neuwied: Luchterhand.

20 Blumler, J. G. and M. Gurevitch, 1995, The Crisis of Public Communication, London/New York: Routledge.

21 Die theoretische Grundlage für diese empirischen Untersuchungen ergibt sich aus der, deliberativen Demokratietheorie', wobei die Variablen ,Interaktivität' und ,kritisch-rationaler Diskurs' als Kernelemente identifiziert und in empirisch messbare Kategorien übersetzt wurden. 
Derzeit arbeiten wir im Rahmen dieser Studie an einer quantitativen Inhaltsanalyse zum Themenbereich ,Europäische Verfassung'. Aus den rund 2.200 Diskussionsthemen mit mehr als 12.000 Beiträgen wurde mittels einer geschichteten Zufallsstichprobe eine repräsentative Stichprobe von rund 600 Postings gezogen. Erste Ergebnisse zeigen, dass fast ein Drittel der vorgeschlagenen Themen keinen Anklang fand. Im Diskursprozess am „Your Voice“-Talkboard zeigt sich, dass die Kommunikationsprozesse meist innerhalb relativ kleiner Gruppen stattfinden; im Allgemeinen erfolgen sie in Englisch, Versuche in den jeweiligen Landessprachen blieben zumeist ohne nennenswerten Erfolg. Ein hoher Grad an rationalen, gut nachvollziehbaren und balancierten Argumenten sowie vielfach hohes Fachwissen deuten darauf hin, dass in diesen Foren ein spezialisiertes (Fach-) Publikum diskutiert. Dies zeigen auch die Themenvorschläge, vielfach grundlegende Problemstellungen $^{22}$ aber auch wichtige aktuelle Themen, wie etwa der Beitritt der Türkei. Erste Ergebnisse lassen daher vermuten, dass dieser Internetdienst der EU adäquate Diskussionsbeziehungen zwischen einigen TeilnehmerInnen fördert, wenngleich zunächst noch die Frage offen bleibt, inwieweit die Talkboard-TeilnehmerInnen solche grundsätzlich qualitativ hochwertigen Diskurse auch in der „offline Welt“ führen, und inwieweit genau die Personen teilnehmen, die sich auch sonst politisch engagieren.

Die Auswirkungen von deliberativen Prozessen auf politische Entscheidungen im Rahmen von Online-Konsultationen wurden empirisch bislang kaum noch erfasst. Mit Hilfe von qualitativen Inhaltsanalysen und ExpertInneninterviews soll im Rahmen unserer Studie im Weiteren die Zufriedenheit der Beteiligten mit dem Konsultationsprozess untersucht, Strategien bzw. Vorgangsweisen zur Umsetzung von BürgerInnenempfehlungen von politischen EntscheidungsträgerInnen erfragt, und die Usability sowie der Zugang zu Informationen erhoben werden; letztlich geht es um die Frage, wieweit der Erosionsprozess politischer Beteiligung mit Hilfe neuer elektronischer Technologien gebremst oder gar umgekehrt werden kann.

\subsection{Das digitale Krankenhaus: Integration einer neuen Technologie in ein bestehendes System}

Geht es bei der e-democracy-Studie somit um den Beitrag einer neuen Technologie zur Lösung eines alten, jedoch zunehmend drängenden Problems, befasste sich unsere Studie über das digitale Krankenhaus ${ }^{23}$ mit der Integration einer neuen Technologie, der digitalen Radiologie, in ein bestehendes komplexes System, nämlich das Krankenhaus. Als maßgebend für die Integration der neuen Technologie in das alte System, wie für die Ausnützung der effizienzsteigernden Potentiale der neuen Technologie erwiesen sich dabei die Schnittstellen, sowohl in technischer als auch in (arbeits-)organisatorischer Sicht.

Obwohl die von uns untersuchte, in Österreich erstmalige Einführung eines digitalen Radiologiesystems (Picture Archiving Communication System - PACS) ein großer Erfolg und in vielfacher Hinsicht beispielgebend war, zeigten sich an den Schnittstellen tatsächlich nicht unerhebliche Probleme. So stellte sich etwa im technischen Bereich heraus, dass die Interoperabilität unterschiedlicher Softwaresysteme trotz internationaler Normung nicht leicht zu realisieren ist: Ergonomie, Akzep-

22 Wie z. B. Sprachendominanz in der EU, Bedeutung von identitätsstiftenden Symbolen wie Flaggen, Hymnen etc., Grund- und Freiheitsrechte in der EU etc.

23 Peissl, W., Tellioglu, H., Wild, C. und Nentwich, M., 1997, Das digitale Krankenhaus - Eine Technikfolgenabschätzung moderner Telekommunikationstechnologien im Krankenhaus am Beispiel des Donauspital/ $S M Z O$, im Auftrag von: BMWVK und Wr. Krankenanstaltenverbund, Wien: Institut für Technikfolgenabschätzung, http://www.oeaw.ac.at/ ita/ebene5/d2-2b9.pdf. 
tanz durch die NutzerInnen und Wirtschaftlichkeit, wie das langfristig angestrebte Ziel einer, elektronischen Krankengeschichte' erfordern eine Integration des neuen elektronischen Radiologie-Bildarchivierungssystems (PACS) in die schon bestehenden Radiologie- und Krankenhaus-Informationssysteme (RIS und KIS); obwohl zwei der drei Systeme von derselben Firma entwickelt und geliefert wurden, war diese Kompatibilität nicht gegeben, ja vom Lieferanten nicht einmal vorgesehen. Umfangreiche und kostspielige Adaptionsarbeiten an den einzelnen Software-Komponenten sowie Ineffizienzen und Störungen im Arbeitsablauf der Station waren die Folge.

Als noch heikler erwies sich die Schnittstelle zwischen Technik und Organisation. Trotz Verwendung standardisierter Komponenten ist eine radiologieinterne Vernetzung ein hochkomplexes System, für dessen Bedienung krankenhausintern System-Know-how aufgebaut werden muss. Die neuen Arbeitsabläufe erfordern Umschichtungen von Personal und z. T. neue Qualifikationen. So steht etwa einer Personaleinsparung im Bereich der Bildverwaltung ein entsprechender Personalbedarf in der Systemwartung gegenüber; die neue Qualifikation einer/s SystemadministratorIn wird erforderlich, die in der täglichen Routine wesentliche Vorteile gegenüber externem Wartungspersonal bringt. Am komplexesten ist jedoch die Neuorganisation der Arbeitsabläufe, die die Stellung und das Selbstverständnis der RadiologInnen im Zusammenspiel der Disziplinen betrifft. Wer befundet die Bilder? Sollen die zuweisenden Ärzte die Bilder sofort, also noch vor der Befundung durch die Röntgenologen, erhalten? Die Autoroutingfunktion, die automatisches Verschicken und mit der Aufnahme zeitgleiches Betrachten des Bildes auch an entfernten Bildschirmen ermöglicht, ändert zumindest die Erwartungshaltung bezüglich Geschwindigkeit der Befundung seitens der KlinikerInnen. Die neue bildgebende Technologie hat einen Wandlungsprozess eingeleitet, dessen Ausgang noch nicht absehbar ist, an dessen Ende aber sicher eine neue Arbeitsteilung zwischen Klinikern und Röntgenologen stehen wird.

Neben Aspekten wie Qualität der Versorgung oder Perzeption der digitalen Radiologie durch die NutzerInnen befasste sich unsere Studie auch mit den rechtlichen Rahmenbedingungen sowie mit Fragen des Datenschutzes und der Datensicherheit. Medizinische Daten sind i. d. R. sensible Daten und unterliegen einem höheren Schutzniveau als ,normale' personenbezogene Daten. Dürfen sie digital übermittelt werden, dürfen Krankengeschichten digital aufbewahrt werden, ist Telemedizin in Form von Ferndiagnose und -behandlung rechtlich zulässig, wer haftet? Die Antworten unserer Studie auf diese Fragen, die von der herrschenden Rechtsmeinung z. T. abweichen, wurden in weiterer Folge vertieft und getrennt publiziert. ${ }^{24}$

\subsection{HTA: Screening vielfach interessengeleiteter Studien}

Mit meinem nächsten Beispiel, Health Technology Assessment, kurz HTA, bleiben wir zwar inhaltlich bei der Medizin, doch aus der Perspektive der Technikfolgen-Abschätzung geht es um etwas ganz anderes. HTA ist eine Methode der Gesundheits-Politikberatung, Evaluation medizinischer Technologien zur Steuerung (Qualitätskontrolle) wie zur Regulierung vor dem Hintergrund steigender Ausgaben für die Gesundheitsversorgung. Unter Health Technologies, den Objekten des Assessments, werden sowohl medizinische Interventionen, Verfahren und Methoden, als auch technische Geräte verstanden. Ziel ist, medizinische Interventionen auf ihre tatsächliche Wirksamkeit, ihre angemessene und effiziente Anwendung, auf Qualitätsveränderungen, klinische und organisatorische Auswirkungen, gesellschaftliche Akzeptanz etc. zu untersuchen und deren Diffusion entsprechend zu steuern.

24 Nentwich, M., 1997, Digitalisierung der Medizin - Zur Klärung einiger rechtlicher Fragen der Telemedizin, Recht der Medizin 4(6), 175-181. 
Die Bedeutung und die rasche Verbreitung von HTA $^{25}$ resultiert aus der problematischen Organisation des Gesundheitswesens: Die Nachfrager sind überwiegend (zwangs-)versichert, und zwar i. d. R. ohne Selbstbehalt, sodass sie nicht an Kosten der Leistung interessiert sind, sondern allein an deren Qualität. Auch die Ärzte und Spitäler sind bloß an der optimalen Behandlung des Patienten interessiert, und es gibt für sie keinen Anreiz zu Kosten-Nutzen-Überlegungen. Anbieter der Gesundheitsleistungen ist ein weitgehend öffentlich finanziertes Gesundheitswesen, das seinerseits Vorleistungen wie Pharmazeutika und medizinische Geräte von einer privatwirtschaftlich organisierten, vielfach oligopolistischen Industrie bezieht. Das ist eine problematische Konstruktion, da diejenigen, die zwischen Kosten und Nutzen abwägen (könnten oder sollten!), nämlich die Ärzte und Spitäler, weder diejenigen sind, die die Kosten tragen (die (Sozial-)Versicherung), noch diejenigen die den Nutzen ziehen (die Patienten). Fast zwangsläufig versuchen daher die Pharmazeutika- und Gerätehersteller Patienten - durch Werbung - und Ärzte - auch durch zusätzliche Maßnahmen zur Anwendung jeweils ihrer Produkte und im Zweifelsfall der teureren Variante zu überreden, selbst wenn letztere keine oder bestenfalls minimale Vorteile bietet. ${ }^{26}$ Diese Vorgehensweise der Industrie liegt vor allem deswegen nahe, weil die Forschungsintensität dieser Branchen hohe Fixkosten bedingt, ${ }^{27}$ sodass der Gewinn der Firmen (z. T. sogar ihr Überleben) weitgehend von einem entsprechenden Absatzvolumen abhängt, vor allem vom Absatzvolumen neuer, noch patentgeschützter und daher teurer Produkte.

Für TA bzw. HTA eröffnet sich unter diesen Voraussetzungen die wichtige Aufgabe, Grundlagen für die Qualitätskontrolle und die Steuerung der Gesundheitsausgaben zu erarbeiten. Zwar gibt es Effizienzuntersuchungen von Pharmazeutika und Geräten in keineswegs geringer Zahl, doch beziehen sich diese vielfach bloß auf eine Wirkungsanalyse eines Produkts oder sie stammen aus dem engeren Umfeld des jeweiligen Produzenten; letzteres vor allem deswegen, weil die diesbezügliche Forschung teuer und ohne Unterstützung durch den Produzenten vielfach gar nicht möglich wäre. Eine der wichtigsten Aufgaben der HTA-Institutionen, mit ihrer Distanz und Unabhängigkeit von allen Interessengruppen - Kostenträgern, Industrie, wie auch der forschenden Medizin -, ist daher ein Screening der vorliegenden Studien, Bewertung ihres Grades an Wissenschaftlichkeit, Ableitung objektiver, intersubjektiv nachvollziehbarer Schlussfolgerungen. Es ist nicht Aufgabe von HTA, wie schon im Zusammenhang mit TA generell betont wurde, abzuschätzen, ob x \% höhere Effizienz der Therapie y \% höhere Kosten rechtfertigt; das ist eine politische Entscheidung. HTA erarbeitet jedoch für solche Entscheidungen die Grundlagen: Wieviel mehr Effizienz, auf welchen Gebieten, bei welchen Anwendungen.

Da es in Österreich kein wie immer geartetes HTA gab, hat ITA in den letzten Jahren - zunächst aus eigenen Mitteln - eine kleine HTA-Gruppe von zunächst zwei Personen aufgebaut, ${ }^{28}$ und für das Entstehen eines entsprechenden Problembewusstseins geworben; Zeit- und Geldmangel verhinderten bisher grundlegende Arbeiten, doch konnten einige Pilotstudien erarbeitet werden, die auch internationale Anerkennung fanden. Ein Thema, mit dem wir uns beschäftigten, waren ausgewählte Probleme der Vorsorgemedizin, speziell Massenscreening versus Konzentration auf Erarbeitung von Indikatoren für ,Risikogruppen-Identifikation'. Diesbezügliche Studien über Genetische Testung

25 In nahezu allen westlichen Ländern arbeiten non-profit Institutionen (derzeit insgesamt 43 Institutionen in 21 Ländern im INAHTA/International Network of HTA Agencies), die häufig in akademischen Institutionen wie etwa an medizinischen Universitäten oder der Gesundheitsadministration nahe stehend angesiedelt sind.

26 Einerseits stehen die Ärzte unter dem Druck ihrer, von der Werbung beeinflussten Kunden, der Patienten, andererseits profitieren sie vielfach von den Marketingmaßnahmen der Pharma- und Gerätehersteller; vielfach sind sie auch als Spezialisten an ,Experimenten' mit neuen Methoden interessiert.

27 D. h. dass die Produktion einer zusätzlichen Packung fast nichts kostet, der Erlös also - von den Werbeund Marketingkosten abgesehen - primär der Deckung der vorangegangen Entwicklungskosten dient.

28 An Konzepten einer Ausweitung, die von der jüngsten Evaluierung dringend empfohlen wurde, und deren Finanzierung wird derzeit gearbeitet. 
bei hereditärem Krebs liegen vor, ${ }^{29}$ an Kolonkarzinom-Screening und Hepatitis C wird gearbeitet. Eine zweite Gruppe von Arbeiten beschäftigt sich mit Therapieformen, die unverhältnismäßig häufig eingesetzt werden und deren ,medizinische Angemessenheit' nicht außer Zweifel steht. Das Projekt: Erythropoietin, ein gentechnologisch hergestelltes Arzneimittel, das bei Tumoranämie in Österreich überdurchschnittlich häufig verwendet wird, konnte abgeschlossen werden. ${ }^{30}$ Eine Studie über ,Monoklonale Antikörper', die für einige kleine Indikationen zugelassen sind, aber viel breiter ,off-label' eingesetzt werden, ist in Arbeit. Als letzte möchte ich diejenigen unserer HTA-Pilotstudien erwähnen, die medizinische Interventionen mit, großen Invarianzen' in Bezug auf die Anwendung bestimmter Therapien betreffen, und bei denen sich daher die Frage nach dem adäquaten Umgang mit diesen stellt. Beispiele sind Cytomegalievirus-Immunglobuline bei Transplantationen und Amphotericin bei Invasiven Aspergillen, die in verschiedenen österreichischen Kliniken in höchst unterschiedlicher Menge verabreicht werden - ohne klar erkennbaren Unterschied im klinischen Ergebnis. ${ }^{31}$

\subsection{Cyberscience: Folgen der Digitalisierung für die Wissenschaft}

In dem letzten Beispiel aus der Arbeit des ITA geht es um ein sehr viel breiteres Thema, nämlich um die Folgen einer Querschnittstechnologie, also einem ganzen Spektrum von Technologien, auf ein enorm breites Feld, nämlich die gesamte Wissenschaft. Das mehrjährige, vom FWF teilfinanzierte ITA-Projekt Die Zukunft der Wissenschaft im Zeitalter der Informations- und Kommunikationstechnologien $^{32}$ hat diese Entwicklungen umfassend aufgearbeitet. Interessant an diesem Thema ist schon die Wechselwirkung der Beeinflussung: Die Informations- und Kommunikationstechnologien und insbesondere das Internet wurden einerseits weitgehend durch die Wissenschaft gestaltet und beeinflussen nun ihrerseits die Wissenschaft: Die bi- und multilaterale direkte Kommunikation wird heute zunehmend über E-Mail abgewickelt; fast alle wissenschaftlichen Zeitschriften erscheinen auch oder z. T. sogar schon ausschließlich elektronisch; Online-Datenbanken und virtuelle Bibliotheken ergänzen oder ersetzen traditionelle Informationsquellen: In Zukunft sind auch verstärkt elektronische Konferenzen und weltweit vernetzte Teamarbeit auf Basis von Groupware-Applikationen zu erwarten. Die traditionelle Wissenschaft entwickelt sich zur „Cyber-Wissenschaft“, zu einer Wissenschaft, die sich zunehmend in einem virtuellen Informations- und Kommunikationsraum abspielt.

Welche neuen Technologien, welche Internetdienste, welche Cyber-Werkzeuge stehen für den Einsatz in der Wissenschaft bereit, welche sind in welchem Ausmaß und wo im Einsatz? Schon die Bestandsaufnahme brachte einige Überraschungen: So etwa zeigte ein Vergleich von dreizehn wissenschaftlichen Disziplinen, dass keineswegs die Naturwissenschaften Hauptnutzerinnen des Internet sind; vor allem die Geschichtswissenschaft ist besonders ,cyber', und die Papyrologie nutzt das Internet in einem bemerkenswerten Ausmaß.

29 Jonas, S., Schamberger, C., Wild, C., 2002, Prädiktive Humangenetische Diagnostik bei hereditärem Mamma- und Kolorektalkarzinom, Wien: Institut für Technikfolgenabschätzung, April.

30 Wild, C., Jonas, S., 2000, Erythropoietin bei Tumoranämie. Ein Assessment, Wien: Institut für Technikfolgenabschätzung, Juni, http://www.oeaw.ac.at/ita/ebene5/d2-2b I5.pdf.

31 Wild, C., Jonas, S., Frank, W., Traunmüller, F., 2001, Aspergillose - Stand des Wissens zu Diagnose, Therapie, Umweltbedingungen - Ein Assessment. Endbericht, Institut für Technikfolgen-Abschätzung, Wien, April 2001, http://www.oeaw.ac.at/ita/ebene5/d2-2b/8.pdf.

32 Nentwich, M., 2003, Cyberscience - Research in the Age of the Internet, Wien: Austrian Academy of Sciences Press. 
Im Zentrum unserer Forschung stand die Frage, welche Auswirkungen der zunehmende Technologieeinsatz auf die Arbeitsweise, die Organisation und letztlich auf die Inhalte der wissenschaftlichen Forschung hat. Die digitale Infrastruktur ist bereits weitgehend vorhanden und hat Veränderungen in den Bereichen Raum-Zeit-Dimension, Informationsbeschaffung, Datenaufbereitung, Kooperation, wie auch Ergebnisdarstellung bewirkt. Die zunächst wohl massivsten Auswirkungen betrafen das wissenschaftliche Publikationswesen. Schon bald werden praktisch alle wissenschaftlichen Publikationen (vielleicht mit Ausnahme der Monographie) nur noch digital erscheinen. Folge sind nicht nur massive Umwälzungen im Verlagswesen; viel bedeutender sind die Folgen für die Archivierung des Wissensbestandes und die Qualitätskontrolle: die neuen Medien sind ja in jeder Weise viel flüchtiger als bedrucktes Papier, in ihrer physischen Existenz wie in der Änderbarkeit ihrer Inhalte. Vermutlich erst am Anfang einer interessanten Entwicklung stehen neue Formen der Wissensdarstellung wie Hypertext, Multimedia oder virtuelle Realität, deren Auswirkungen auf die Weiterverarbeitung von wissenschaftlichen Wissen und auf dessen Produktion erst ansatzweise abschätzbar sind. Bezüglich der Folgen der Digitalisierung für die Inhalte der Forschung vermutet die Studie gravierende Auswirkungen in vier Dimensionen: Als Folge methodischer Neuerungen, etwa des , verteilten Rechnens', als Folge veränderter Arbeitsweisen, insbesondere durch Beschleunigungseffekte, als Folge von Vorwirkungen der neuen Darstellungsformen, etwa der Aufbereitung von Wissen für Hypertextdatenbanken, und schließlich als Folge des Computers als Werkzeug an sich, etwa bei Simulationen oder durch den Einsatz Künstlicher Intelligenz.

Handlungsbedarf als Folge der Digitalisierung der Wissenschaft diagnostiziert die Studie etwa in Bezug auf die Bereitstellung der erforderlichen technischen Infrastruktur und die Gewährleistung eines allgemeinen Zugangs zu dieser, in Bezug auf Fragen des Rechts des geistigen Eigentums im Internet, in Bezug auf die Sicherstellung der Qualität der digitalen Bestände und ihrer nachhaltigen Archivierung, sowie in Bezug auf die Ausbildung der Bibliothekare und Forschenden. Entscheidend für die zukünftige Entwicklung dürfte die Frage der Kommerzialisierung bzw. Entkommerzialisierung des wissenschaftlichen Veröffentlichungswesens sein, da die Digitalisierung wissenschaftlicher Information Potenzial zum freien Zugang wie zur weitgehenden Abschottung in sich birgt - die Tendenzen zu letzterer scheinen derzeit vor allem im Bereich der Naturwissenschaften bedauerlicherweise zu überwiegen. Handlungsbedarf sollte allerdings nicht bloß bei der Politik gesehen werden, angesprochen sind neben staatlichen und überstaatlichen Einrichtungen auch Forschungsbibliotheken, Universitäten, Forschungseinrichtungen und wissenschaftliche Fachgesellschaften.

Das Projekt „Cyberscience“ ist ein Beispiel für eine umfassende Aufbereitung und Analyse des Technikeinsatzes in einem ganzen Gebiet; es generiert Überblickswissen, diagnostiziert künftige Entwicklungen ihrer Art nach, deckt relevante Themen und potentiellen Handlungsbedarf auf. Eine Aussage, ob der Einsatz des Internet in der Wissenschaft insgesamt gut oder schlecht sei, ist natürlich jenseits des Aufgabenbereichs von TA. 


\section{Resümee}

Lassen Sie mich, sehr geehrte Damen und Herren, zum Eingangssatz zurückkehren: Es gäbe keine Technik, die durch Technikfolgen-Abschätzung ermöglicht oder verhindert worden wäre. Der Satz ist richtig: Es gibt tatsächlich keine. Ich hoffe aber, Sie davon überzeugt zu haben, dass es die Förderung oder Verhinderung von bestimmten Techniken gar nicht Aufgabe von TA sein kann. Technikfolgen-Abschätzung hat andere, vielleicht weniger plakative aber dennoch wichtige Aufgaben. Ich habe versucht, Ihnen das an Hand folgender Beispiele zu unserer Arbeit zu zeigen:

- Aufspüren der Motive und der tieferliegenden Ursachen der Vorbehalte breiter Kreise der Bevölkerung gegenüber der Grünen Gentechnik,

- Erforschung der kulturellen Leitbilder, an denen sich die Debatte um die Humangenetik orientiert,

- Untersuchung der On-Line-Debatten zu EU-Problemen auf ihr Potential, der Erosion demokratischer Partizipation entgegen zu wirken, als Ausleuchtung des Potentials einer spezifischen Technologie-Anwendung,

- Analyse der Schnittstellenprobleme bei der Integration einer neuen Technologie, der digitalen Radiologie, in ein bestehendes System,

- Screening vielfach interessen-geleiteter Studien im Rahmen des Health Technology Assessment als Basis für gesundheitspolitische Entscheidungen, und letztlich

- die Folgen der Digitalisierung im weitesten Sinn für die Entwicklung der Wissenschaft.

Ich hoffe, dass meine Beispiele aus der Arbeit des Instituts für Technikfolgen-Abschätzung zumindest andeuten konnten, dass wir tatsächlich einige Beiträge zur Verbesserung der Entscheidungsgrundlagen für wichtige Fragen geliefert haben. 


\section{Anhang A: Das Institut für Technikfolgen- Abschätzung der ÖAW}

Das ITA ist schon von der Struktur seiner Mitarbeiter multidisziplinär angelegt: Diese decken die Disziplinen Recht, Soziologie, Betriebswirtschaft, Volkswirtschaft, Publizistik- und Kommunikationswissenschaft, Ökologie, Biotechnologie, Medizin, Verfahrenstechnik, Nachrichtentechnik und Wissenschaftsforschung ab; je nach Aufgabenstellung wird dieses Team durch auswärtige Experten verstärkt. Als relativ kleines Institut konzentriert sich das ITA auf die Gebiete Biotechnologie, Informations- und Kommunikationstechnologie, Medizintechnologie und Umwelttechnologie und Umwelt; andere Bereiche, wie etwa Verkehr und Energie müssen ausgespart bleiben, aus Kapazitätsgründen, aber auch weil diese Gebiete in Österreich durch andere Institute abgedeckt werden.

Die im Verlaufe dieser Studie besprochenen Arbeiten wurden unter dem Gesichtspunkt ihrer Repräsentativität für die Aufgaben von TA ausgewählt; insofern zeigen sie bloß einen speziellen Ausschnitt aus der Arbeit des ITA. Anhang B bietet daher eine Aufstellung der Arbeiten der letzten fünf Jahre und der laufenden Projekte. 


\section{Anhang B: Die Arbeiten der letzten fünf Jahre}

\begin{tabular}{|c|c|c|}
\hline Titel & Laufzeit & Finanzierung \\
\hline \multicolumn{3}{|l|}{ Biotechnologie } \\
\hline $\begin{array}{l}\text { Das post-genomische Zeitalter: Wie verändert die zunehmende } \\
\text { Komplexität die Debatte über die Genetik? (POCO) }\end{array}$ & 03/2004-09/2006 & GenAU \\
\hline $\begin{array}{l}\text { Partizipative Politikberatung am Beispiel der Bürgerlnnen- } \\
\text { konferenz } 2003 \text { (Analyse) }\end{array}$ & $10 / 2003-05 / 2004$ & RFT \\
\hline $\begin{array}{l}\text { Vorsorgende Expertise bei genetisch veränderten } \\
\text { Kulturpflanzen }\end{array}$ & $03 / 2002-03 / 2004$ & EU (5. RP) \\
\hline Life Science in der europäischen Gesellschaft (LSES) & $02 / 2000-02 / 2003$ & EU (5. RP) \\
\hline $\begin{array}{l}\text { Gentechnik und Demokratie - Der österreichische } \\
\text { Gentechnik-Konflikt im internationalen Zusammenhang }\end{array}$ & $01 / 1999-06 / 2001$ & ITA \\
\hline $\begin{array}{l}\text { Die wissenschaftliche Basis der Anwendung des } \\
\text { Vorsorgeprinzips in drohenden Handelskonflikten im } \\
\text { Zusammenhang mit Biotechnologie }\end{array}$ & I I/2000-05/200I & EU (ESTO) \\
\hline $\begin{array}{l}\text { Der europäische Diskurs zum Thema Biotechnologie: } \\
\text { Dimensionen der öffentlichen Betroffenheit (EUDEB) }\end{array}$ & $01 / 1999-12 / 2000$ & EU (5. RP) \\
\hline $\begin{array}{l}\text { Österreichischer Beitrag zu einem Teil des OECD-Berichts } \\
\text { an die G8 über „Biotechnologie und andere Aspekte der } \\
\text { Lebensmittelsicherheit“" }\end{array}$ & $12 / 1999-05 / 2000$ & UBA \\
\hline \multicolumn{3}{|l|}{ Informations- und Kommunikationstechnologie } \\
\hline Gesellschaftliche Grundlagen des Rechts auf Privatsphäre & $05 / 200 I-12 / 2004$ & ITA \\
\hline Online Debatten und Konsultationen in der Europäischen Union & $06 / 2003-11 / 2004$ & BMBWK (NODE) \\
\hline $\begin{array}{l}\text { Datenschutz durch Technik: Privacy Enhancing Technologies } \\
\text { im Test }\end{array}$ & $03 / 2002-06 / 2004$ & ITA \\
\hline $\begin{array}{l}\text { Europäische Wissensdatenbank über sozio-ökonomische } \\
\text { Folgenabschätzungen der Technologien für die } \\
\text { Informationsgesellschaft (EKB-SEIS) }\end{array}$ & $06 / 2003-02 / 2004$ & EU (ESTO) \\
\hline $\begin{array}{l}\text { Reorganisation von „Back-Offices“ in der Verwaltung in } \\
\text { Hinblick auf die Verbesserung öffentlicher Dienstleistungen } \\
\text { (BACKOFFICE) }\end{array}$ & $03 / 2003-12 / 2003$ & EU (5. RP) \\
\hline Regierung und Demokratie im Informationszeitalter (COST AI4) & $03 / 1998-06 / 2003$ & EU (COST AI4) \\
\hline $\begin{array}{l}\text { Entwicklung und Bewertung innovativer Dienstleistungsmodelle } \\
\text { (PRISMA) }\end{array}$ & $09 / 2000-04 / 2003$ & EU (5. RP) \\
\hline $\begin{array}{l}\text { Die Zukunft der Wissenschaft im Zeitalter der Informations- } \\
\text { und Kommunikationstechnologien }\end{array}$ & $10 / 1998-03 / 2003$ & FWF, ITA \\
\hline Datenvermeidung in der Praxis & $02 / 2002-06 / 2002$ & AK Wien \\
\hline $\begin{array}{l}\text { Ubiquitous Computing: Entwicklungszeiträume, Stärken/ } \\
\text { Schwächen Europas bei Basistechnologien, technologie } \\
\text { politischer Handlungsbedarf }\end{array}$ & $09 / 2000-03 / 2001$ & EU (ESTO) \\
\hline Wettbewerb bei Zugangsnetzen & $10 / 1999-12 / 2000$ & ITA \\
\hline Beeinträchtigungen der Privatsphäre in Österreich & $09 / 2000-10 / 2000$ & AK Wien \\
\hline One-stop Government in Österreich & $05 / 1999-12 / 1999$ & EU (COST AI4) \\
\hline $\begin{array}{l}\text { Elektronische Informationsdienste auf Bundesebene in } \\
\text { Österreich (e-GOVERNMENT I) }\end{array}$ & $01 / 1999-07 / 1999$ & BKA \\
\hline
\end{tabular}




\begin{tabular}{|c|c|c|}
\hline Titel & Laufzeit & Finanzierung \\
\hline \multicolumn{3}{|l|}{ Medizintechnologie } \\
\hline $\begin{array}{l}\text { Chronische Hepatitis C - Implikationen für Therapie und } \\
\text { ökonomischen Ressourceneinsatz in Österreich }\end{array}$ & $01 / 2004-06 / 2004$ & AESCA, ITA \\
\hline Asthma in der Kindheit & $02 / 2004-04 / 2004$ & EU (ESTO) \\
\hline $\begin{array}{l}\text { Flexible Sigmoidoskopie: Implementierungsmöglichkeiten } \\
\text { und Grenzen eines Kolon-Karzinom Screenings - unter } \\
\text { besonderer Berücksichtigung der flexiblen Sigmoidoskopie vs. } \\
\text { Kolonoskopie in Österreich }\end{array}$ & $09 / 2003-12 / 2003$ & HVdÖSVT \\
\hline $\begin{array}{l}\text { Nicht-ionische Röntgenkontrastmittel - Analyse zur klinischen } \\
\text { Relevanz der unterschiedlichen Röntgenkontrastmittel }\end{array}$ & $07 / 2003-11 / 2003$ & Wiener KAV, ITA \\
\hline Evidenzbasierte Bedarfsplanung für Intensivbetten & $01 / 2002-12 / 2002$ & TILAG, ITA \\
\hline $\begin{array}{l}\text { Prädiktive humangenetische Diagnostik bei hereditären Krebs- } \\
\text { erkrankungen. Schwerpunkte: Mamma- und Ovarialkarzinom, } \\
\text { Kolorektalkarzinom }\end{array}$ & $10 / 200 \mid-03 / 2002$ & $\begin{array}{l}\text { Fonds Gesundes } \\
\text { Österreich, ITA }\end{array}$ \\
\hline $\begin{array}{l}\text { Medizinische Effektivität von Glycoprotein Ilb/llla Rezeptor- } \\
\text { antagonisten in der Therapie von akuten Koronarsyndromen }\end{array}$ & $10 / 2001-03 / 2002$ & $\begin{array}{l}\text { Bundeskasse } \\
\text { Bonn, ITA }\end{array}$ \\
\hline $\begin{array}{l}\text { Immunglobuline in der Prävention/Therapie der Zytomegalie- } \\
\text { Virusinfektion nach Transplantationen }\end{array}$ & $0 \mathrm{I} / 200 \mathrm{I}-09 / 200 \mathrm{I}$ & $\begin{array}{l}\text { Klinikum Graz, } \\
\text { ITA }\end{array}$ \\
\hline Früherkennung der Aspergillose & ||$/ 2000-06 / 200 \mid$ & TILAK, ITA \\
\hline Koordinierung und Vernetzung von HTA (ECHTA) & 07/1999-04/2001 & ITA \\
\hline HTA-Evaluationsmethoden in Europa (ASTEC) & $01 / 1999-08 / 2000$ & ITA \\
\hline Erythropoietin bei Tumoranämie & $01 / 2000-07 / 2000$ & ITA \\
\hline $\begin{array}{l}\text { CRP-Messung bei niedergelassenen Ärztlnnen: Eine Chance } \\
\text { zur Reduktion der Antibiotikaverschreibungen? }\end{array}$ & $01 / 2000-07 / 2000$ & ITA \\
\hline \multicolumn{3}{|l|}{ Umwelttechnologie und Umwelt } \\
\hline $\begin{array}{l}\text { Politische Wege zur Förderung der Entwicklung und Einsatz } \\
\text { von Cleaner Technologies (POPA) }\end{array}$ & $01 / 2004-12 / 2005$ & EU (6. RP) \\
\hline $\begin{array}{l}\text { Bewertung der Folgen von Luftemissionen von Emerging } \\
\text { Technologies }\end{array}$ & $01 / 2004-12 / 2004$ & EU (ESTO) \\
\hline Strategische Umweltprüfung & 01/1996-Offen & ITA, ÖAW-Verlag \\
\hline Diffusion von industriellen clean technologies & $1 \mid / 2002-12 / 2003$ & EU (ESTO) \\
\hline $\begin{array}{l}\text { Bewertung innovativer Technologien im Umweltbereich im } \\
\text { Rahmen des Leitbildes Nachhaltigkeit: Fallbeispiel: Grüne } \\
\text { Bioraffinerie }\end{array}$ & $10 / 2001-06 / 2003$ & ITA \\
\hline $\begin{array}{l}\text { Anforderungen an Methoden zur Bewertung innovativer } \\
\text { komplexer Technologien am Beispiel biologisch abbaubarer } \\
\text { Polymere }\end{array}$ & 08/2000-08/200I & BMU \\
\hline $\begin{array}{l}\text { Bewertung des Durchsetzungspotentials und der } \\
\text { Wirtschaftlichkeit vorsorgender Technologien }\end{array}$ & II/I999-06/2000 & BMU \\
\hline $\begin{array}{l}\text { Anforderungen zur Verbesserung der „Öko-Effizienz“ im } \\
\text { Chemiesektor }\end{array}$ & 04/1999-06/1999 & EU (IPTS) \\
\hline
\end{tabular}




\section{Bisher erschienene manu:scripte}

ITA-0I-0I Gunther Tichy, Walter Peissl (12/2001): Beeinträchtigung der Privatsphäre in der Informationsgesellschaft. <http://www.oeaw.ac.at/ita/pdf/ita_0I_OI.pdf>

ITA-0I-02 Georg Aichholzer(12/2001): Delphi Austria: An Example of Tailoring Foresight to the Needs of a Small Country. <http://www.oeaw.ac.at/ita/pdf/ita_0I_02.pdf>

ITA-0I-03 Helge Torgersen, Jürgen Hampel (12/2001): The Gate-Resonance Model: The Interface of Policy, Media and the Public in Technology Conflicts.

<http://www.oeaw.ac.at/ita/pdf/ita_0I_03.pdf>

ITA-02-0I Georg Aichholzer (01/2002): Das ExpertInnen-Delphi: Methodische Grundlagen und Anwendungsfeld ,Technology Foresight“.

<http://www.oeaw.ac.at/ita/pdf/ita_02_0l.pdf>

ITA-02-02 Walter Peissl (01/2002): Surveillance and Security - A Dodgy Relationship.

<http://www.oeaw.ac.at/ita/pdf/ita_02_02.pdf>

ITA-02-03 Gunther Tichy (02/2002): Informationsgesellschaft und flexiblere Arbeitsmärkte. $<$ http://www.oeaw.ac.at/ita/pdf/ita_02_03.pdf $>$

ITA-02-04 Andreas Diekmann (06/2002): Diagnose von Fehlerquellen und methodische Qualität in der sozialwissenschaftlichen Forschung.

$<$ http://www.oeaw.ac.at/ita/pdf/ita_02_04.pdf >

ITA-02-05 Gunther Tichy (10/2002): Over-optimism Among Experts in Assessment and Foresight. $<$ http://www.oeaw.ac.at/ita/pdf/ita_02_05.pdf >

ITA-02-06 Hilmar Westholm (12/2002): Mit eDemocracy zu deliberativer Politik? Zur Praxis und Anschlussfähigkeit eines neuen Mediums.

$<$ http://www.oeaw.ac.at/ita/pdf/ita_02_06.pdf $>$

ITA-03-0I Jörg Flecker und Sabine Kirschenhofer (01/2003): IT verleiht Flügel? Aktuelle Tendenzen der räumlichen Verlagerung von Arbeit. $<$ http://www.oeaw.ac.at/ita/pdf/ita_03_0l.pdf>

ITA-03-02 Gunther Tichy (11/2003): Die Risikogesellschaft - Ein vernachlässigtes Konzept in der europäischen Stagnationsdiskussion.

$<$ http://www.oeaw.ac.at/ita/pdf/ita_03_02.pdf>

ITA-03-03 Michael Nentwich (11/2003): Neue Kommunikationstechnologien und Wissenschaft - Veränderungspotentiale und Handlungsoptionen auf dem Weg zur Cyber-Wissenschaft. <http://www.oeaw.ac.at/ita/pdf/ita_03_03.pdf>

ITA-04-0I Gerd Schienstock (1/2004): Finnland auf dem Weg zur Wissensökonomie - Von Pfadabhängigkeit zu Pfadentwicklung. <http://www.oeaw.ac.at/ita/pdf/ita_04_0I.pdf>

ITA-04-02 Gunther Tichy (6/2004): Technikfolgen-Abschätzung: Entscheidungshilfe in einer komplexen Welt. <http://www.oeaw.ac.at/ita/pdf/ita_04_02.pdf> 